\title{
A Fuzzy GM(1,1) Model Based Possibility Check for Predicting $\mathrm{CO}_{2}$ Emissions
}

\author{
Zhiyi Meng
}

\begin{abstract}
Increasing emissions of $\mathrm{CO}_{2}$ and other greenhouse gases as a result of human activities have bring about global warming, which is one of the major threats now confronting the environment. $\mathrm{CO}_{2}$ have impact on the environment is the greatest as it accounts for the largest share of total GHG. The greenhouse effect will further destroy the environment for humans and all other living beings, threatening the existence of humankind if anthropogenic $\mathrm{CO}_{2}$ emissions are allowed to increase without limits. The reduction of $\mathrm{CO}_{2}$ emissions has become a key issue that must be addressed for the protection of the environment. This paper aims to develop a fuzzy gray prediction model on $\mathrm{CO}_{2}$ emissions, and the world natural and cultural heritage area will be taken as an example. The fuzzy possibility is used to check the error of the proposed model, and the test results show that the accuracy of the model is quite high, providing a scientific basis for policy makers.
\end{abstract}

Index Terms $-\mathrm{CO}_{2}$ emission, fuzzy gray prediction, world natural and cultural heritage area, fuzzy possibility.

\section{INTRODUCTION}

Increasing emissions of $\mathrm{CO}_{2}$ and other greenhouse gases as a result of human activities have brought about global warming, which is one of the major threats now confronting the environment [1]. $\mathrm{CO}_{2}$ have impact on the environment is the greatest as it accounts for the largest share of total GHG [2]. The greenhouse effect will further destroy the environment for humans and all other living beings [3], threatening the existence of humankind if anthropogenic $\mathrm{CO}_{2}$ emissions are allowed to increase without limits. To achieve $\mathrm{CO}_{2}$ emissions reduction, the first thing to do is to acquire accuracy quantity and trend of $\mathrm{CO}_{2}$ emissions in global and regional scope. In other words, we should make a prediction on the $\mathrm{CO}_{2}$ emissions in the next few years to guarantee the decision makers to take scientific and effective measures.

The city chosen in this paper is the world natural and cultural heritage area, Leshan, in Western China, which is an ancient city with three thousand years of history. The Leshan Giant Buddha is the largest seated stone Buddha in the world and Mount Emei is a mountain scenic area that contains both Buddhist culture and natural beauty. In 1996, the Leshan Giant Buddha-Mount Emei region was recognized as a world natural and cultural heritage area. However, the carbon

Manuscript received September 20, 2014; revised November 21, 2014 This research was supported by the Key Program of NSFC (Grant No. 70831005), and the Key Project of China Petroleum and Chemical Corporation (Grant No. GJ-73-0706).

Zhiyi Meng is with the Uncertainty Decision-Making Laboratory, Sichuan University, Chengdu 610064, China (e-mail zhiyimengscu@sina.com). intensity in this region has increased quickly from 0.51 in 2005 year to 0.71 in 2008 year, which reflects the environment protection of the world natural and cultural heritage facing a great challenge.

Previously, various studies predicted $\mathrm{CO}_{2}$ in many ways. King [4] predicted regional $\mathrm{CO}_{2}$ exchange between the atmosphere and the terrestrial biosphere by using ecosystem models. Bacastow and Keeling [5] discuss the models to predict future atmospheric $\mathrm{CO}_{2}$ concentrations. Raich [6] studies global patterns of carbon dioxide emissions from soils. Kim [7] focus on solubility measurement and prediction of carbon dioxide in ionic liquids. Park [8] researched correlation and prediction of the solubility of carbon dioxide in aqueous alkanolamine and mixed alkanolamine solutions. However, there are few studies predicted $\mathrm{CO}_{2}$ emissions use $\operatorname{GM}(1,1)$ model under fuzzy environment.

Grey theory, developed originally by Deng [9], is a truly multidisciplinary and generic theory that deals with systems that are characterized by poor information or for which information is insufficient. The fields covered by grey theory include systems analysis, data processing, modeling, prediction, decision making and controlling. The grey theory mainly works on systems analysis with poor, incomplete or uncertain messages. Grey forecasting models have been extensively used in many applications. Furthermore, there are many uncertainties when setting the objectives. Using one kind of variable to describe those uncertainties is very important. Thus, a fuzzy variable is considered. Since Zimmermann [10] first introduced conventional linear programming and multi-objective linear programming into fuzzy set theory, there is now an overwhelming amount of research on using fuzzy method to solve uncertainty problem. However, combing $\operatorname{GM}(1,1)$ with fuzzy method to provide support for decision makers is absent from current research.

In addition, the data of $\mathrm{CO}_{2}$ emissions is locked and inaccuracy, policy makers' high demand for reliable model results has not yet been fulfilled. In this paper, fuzzy grey method will be employed to make prediction on emissions in the world nature and culture heritage areas. The rest of the paper is organized as follows. Section II presents the general fuzzy gray prediction model and discusses th $\mathrm{CO}_{2} \mathrm{e}$ model checking method. Section III discusses the use of fuzzy gray prediction method and its application on $\mathrm{CO}_{2}$ emissions in Leshan city, besides analysis and corresponding countermeasures and measures also presents in Section IV. Finally, conclusions are presented in Section IV.

\section{METHODOLOGY}

This part mainly includes the following points. Firstly, of 
the $\mathrm{CO}_{2}$ emissions in Leshan city is basically introduced. Secondly, fuzzification method is described. In addition, the Grey prediction method is shown here as well as the fuzzy possibility check method.

\section{A. Basic Introduction}

Fig. 1 shows the $\mathrm{CO}_{2}$ emissions in Leshan city. From 2000 to 2011 , the emissions in Leshan city has increased to $1305845 \mathrm{~T}$ from $355742 \mathrm{~T}$, the change is overwhelming, which multiplies pressure for environmental protection in world natural and cultural heritage areas. In order to make scientific decisions, policymakers need to have a specific understanding of emissions in Leshan city. So, predict the emissions accurately are of great importance for environmental protection in the world natural and cultural heritage areas.

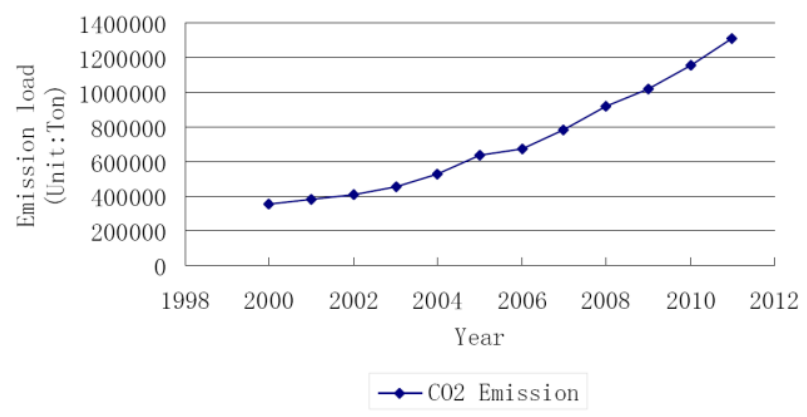

Fig. 1. The $\mathrm{CO}_{2}$ emissions of Leshan city in $2000 \sim 2011$.

As the level of detection technology and means of $\mathrm{CO}_{2}$ is limited, in the process of detecting $\mathrm{CO}_{2}$, some factors cannot be accurately measured, so the results are often displayed in an estimate. If we directly make predictions based on this set of data then the data obtained is obviously inaccurate. So, before we predict, we should deal with the original data. In this paper, the basic theory of fuzzy will be used to make fuzzification on original data, then we will make predictions based on the fuzzy data. This model is targeted at trends of the emissions. It deals with issues of combining global and local constraints various actors are at stake. The main variables and the influential factors of the model are identified on the base which refers to the relevant information worldwide, as well as the analysis of the other related factors. This model consists of two parts: 1) Fuzzification 2) Grey prediction. Here, we first introduce the basic theory of Fuzzification. The whole process of the modeling is shown in Fig. 2.

\section{B. Fuzzification}

It is difficult to detect the $\mathrm{CO}_{2}$ for its own characteristics. As mentioned, before detection techniques and tools for $\mathrm{CO}_{2}$ emissions levels is limited, which lead to some errors in the data collected. So, if we ignore these errors, and analyze these data and make predictions based on them, then the accuracy and science of the results obtained will be reduced. In this paper, we first make fuzzification based on the original data.

Fuzzy set theory has been developed to solve problems where the descriptions of activities and observations are imprecise, vague, or uncertain. The term "fuzz" refers to a situation in which there are no well defined boundaries of the set. To cope with this difficulty, Zadeh [11] proposed the fuzzy set theory. A fuzzy set is regarded as a class of objects with a continuum of membership grades. A membership function, which assigns to each object a grade of membership, is associated with each fuzzy set. Usually, the membership grades are in the interval $[0,1]$. When the grade of membership for an object in a set is one, this object is absolutely in that set; when the grade of membership is zero, the object is absolutely not in that set. Borderline cases are assigned numbers between zero and one. Precise membership grades do not convey any absolute significance as they are context-dependent and can be subjectively assessed.

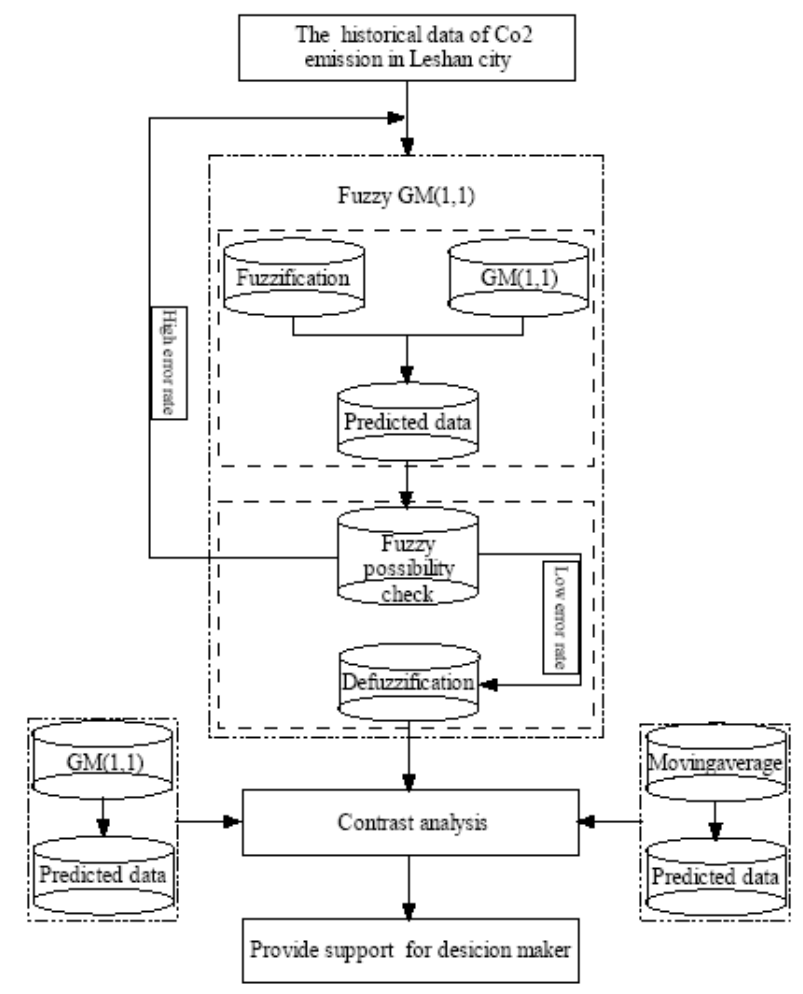

Fig. 2. Modeling process of Fuzzy Grey $(1,1)$

Sometimes, we might only need object of a fuzzy set instead of its characteristic function, that is, to transfer a fuzzy set into a crisp set. In order to do so, we need one concept, $\alpha$-cut. It is often necessary to consider those elements in a fuzzy set which have nonzero membership grades. These elements are the support of that fuzzy set. Zadeh regards that the $\alpha$-cut for a fuzzy set, A should be defined as

$$
A \alpha=\left\{x \mid \mu_{A}(x) \geq 0 \text { and } \mathcal{X} \in U\right\}
$$

The term fuzzy number is used to handle imprecise numerical quantities. A general definition of a fuzzy number is given that any fuzzy subset $H=\{(x, \mu(x))\}$, where $\mathrm{x}$ take number on the real line $\mathrm{R}$ and $\mu_{H}(x) \in[0,1]$. Here, we use the basic theory of triangular fuzzy numbers proposed by Van [20]. He claimed that a triangular fuzzy number $\mathrm{H}$ can be defined by a triplet $\left(Q^{-}, Q, Q^{+}\right)$(The character $Q$ stands for $\mathrm{CO}_{2}$ in the following text). Its membership function is shown in Fig. 3. The whole process of fuzzification can be summarized as follows.

Step 1: Calculated the left variation $\alpha$ and the right variation $\beta$ according to the empirical data. 
Step 2: Determine the fuzzy interval $\left[Q^{-}, Q^{+}\right]$according to the left and right variations, where $Q^{-}=Q-\alpha$ and $Q^{+}=Q+\beta$.

Step 3: Construct the membership function $\mu(x)$ such that $\mu(Q)=1$ according to experts' suggestion.

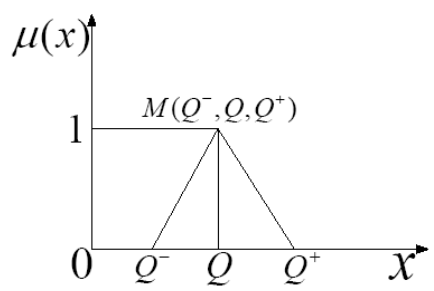

Fig. 3. The membership function of a triangular fuzzy number.

Step 4: Define the fuzzy time series. It can be expressed as follows. $\left(\left[Q_{1}, \mu_{1}(x)\right] \$, \$\left[Q_{2}, \mu_{2}(x)\right], \cdots,\left[Q_{m}, \mu_{m}(x)\right]\right)$, where $Q_{i}$ is the empirical value of $\mathrm{CO}_{2}$ emissions according to the survey and $\mu_{i}(x)$ is the membership function experts suggested on the $i$ th year.

\section{Grey Prediction}

The GM(1,1) forecasting model adopts the essential part of grey system theory and it has been successfully used in finance, integrated circuit industry and the market for air travel. The advantages of the grey model are: (1) it can use only a few data to estimate an unknown system, and (2) it can use a first order differential equation to characterize the unknown system behavior [12].

In contrast to statistical methods, the potency of the original series in the time series grey model, called $\operatorname{GM}(1,1)$, has been proved to be more than four. In addition, assumptions regarding the statistical distribution of data are not necessary when applying grey theory. The accumulated generation operation (AGO) is one of the most important characteristics of grey theory, and its main purpose is to reduce the randomness of data. In fact, functions derived from AGO formulations of the original series are always well fitted to exponential functions.

Set the original value of $\mathrm{CO}_{2}$ emissions as a group of original sequence $Q^{(0)}$, having observing the datum.

Step 1: Set the original value of $\mathrm{CO}_{2}$ emissions as a group of original sequence $Q^{(0)}$,

$$
Q^{(0)}=\left(Q^{(0)}(1), Q^{(0)}(2), Q^{(0)}(3)\right), \cdots,\left(Q^{(0)}(n)\right) .
$$

Step 2: A new sequence $Q^{(1)}$ is generated by the accumulated generating operation (AGO),

$$
Q^{(1)}=\left(Q^{(1)}(1), Q^{(1)}(2), Q^{(1)}(2)\right), \cdots,\left(Q^{(1)}(n)\right) .
$$

where $Q^{(1)}(k)=\sum_{i=1}^{k} Q^{(1)}(i)$

Step 3: Establishing a first-order differential equation.

$$
\left(\frac{d Q^{(1)}}{d t}\right)+a Z=u
$$

where,

$$
Z^{(k)}=\alpha Q^{(1)(k)}+(1-\alpha) Q^{(1)(k+1)}, k=1,2, \cdots, n-1 .
$$

$\alpha$ denotes a horizontal adjustment coeffcient, and $0<\alpha<1$. The selecting criterion of $\alpha$ value is to yield the smallest forecasting error rate.

Step 4: Form Step 3, we have

$$
Q^{(1)}(k+1)=\left(Q^{(0)}(1)-\frac{u}{a}\right) e^{-a k}+\frac{u}{a}
$$

where

$$
\begin{gathered}
\hat{\theta}=\left[\begin{array}{l}
a \\
u
\end{array}\right]=\left(B^{T} B\right)^{-1} B^{T} Y, B=\left[\begin{array}{cc}
-z^{1}(2) & 1 \\
-z^{1}(3) & 1 \\
\cdots & \cdots \\
-z^{1}(n) & 1
\end{array}\right] \\
Y=\left(\left(Q^{(0)}(2), Q^{(0)}(3), Q^{(0)}(4)\right), \cdots,\left(Q^{(0)}(n)\right)^{T} .\right.
\end{gathered}
$$

Step 5: Inverse accumulated generation operation (IAGO). Because the grey forecasting model is formulated using the data of AGO rather than original data, IAGO can be used to reverse the forecasting value. Namely

$$
Q^{(0)}(k)=\left(Q^{(1)}(k)-Q^{(1)}(k-1)\right), k=2,3, \cdots, n .
$$

\section{Fuzzy Possibility Check}

Input the $\mathrm{CO}_{2}$ emissions data of the past 12 years in Leshan city into the fuzzy grey prediction model, we get a group of forecast data and a prediction curve. In order to view the fitting degree between the forecast data and historical data, we have to test the fuzzy grey forecasting model. Here, fuzzy possibility is used to check the accuracy of the model, and some related theories will be introduced as follows.

Definition 1. Assume $\tilde{M}, \tilde{N}$ as two fuzzy numbers,to any binary operations, membership function of fuzzy number is defined as:

$$
\mu_{\tilde{M}(*) \tilde{N}}(z)=\sup _{x, y: z=x * y} \min \left\{\mu_{\tilde{M}}(x), \mu_{\tilde{N}}(y)\right\}
$$

If $\tilde{M}, \tilde{N}$ still the triangular fuzzy number, that means $\tilde{M}=\left(m_{L}, m, m_{R}\right), \tilde{N}=\left(n_{L}, n, n_{R}\right)$, then the sum, difference of them also is triangular fuzzy number, exist:

$$
\begin{gathered}
\tilde{M}(+) \tilde{N}=\left(m_{L}+n_{L}, m+n, m_{R}+n_{R}\right) \\
\tilde{M}(-) \tilde{N}=\left(m_{L}-n_{R}, m-n, m_{R}-n_{L}\right) .
\end{gathered}
$$

Definition 2. Let $u \in E^{1}$. Since the class of closed intervals $\left\{[v]^{r}=\left\{|\lambda|: \lambda \in[u]^{r}, r \in[0,1]\right\}\right\}$ determines a unique fuzzy number, we denote this fuzzy number as $|\mathrm{u}|$, and call it to be the absolute value of $\mathrm{u},[|u|]^{r}=\left[|u|_{r}^{-},|u|_{r}^{+}\right]$, where, 


$$
\begin{aligned}
& |u|_{r}^{-}=\max \left\{\frac{1}{2}\left(u_{r}^{-}+|u|_{r}^{-}\right), \frac{1}{2}\left(|u|_{r}^{+}-u_{r}^{+}\right)\right\}, \\
& |u|_{r}^{+}=\max \left\{\left|u_{k}^{-}\right|,\left|u_{r}^{+}\right|\right\}
\end{aligned}
$$

\section{Error checking process:}

Let $Q_{i}=\left(a_{i}, b_{i}, c_{i}\right)(i=2 \cdots 9)$ be a triangular fuzzy number such that $0<a_{i}<b_{i}<c_{i}, Q_{i}=\left(a_{i}, b_{i}, c_{i}\right)\left(0<a_{i}<b_{i}<c_{i}\right) \quad$ be the predicted value which is also a triangular fuzzy number. If $\bar{f}$ is the error the decision maker permits. Then we can check the by the formula

$$
\operatorname{POS}\left(\left|Q_{i}-Q_{i}\right| \leq f_{i}\right) \geq \alpha_{i}
$$

Since $Q_{i}$ and $Q_{i}$ are both triangular fuzzy numbers, it follows Definition 1 that $Q_{i}-Q_{i}$ is also a fuzzy number satisfying that

$$
Q_{i}-Q_{i}=\left(a_{i}-c_{i}, b_{i}-b_{i}, c_{i}-a_{i}\right),
$$

according to the definition of $\alpha$-cut set, we have

$$
\begin{aligned}
& \left(Q_{i}-Q_{i}\right)_{\alpha}^{-}=\alpha_{i}\left(b_{i}-b_{i}\right)+\left(1-\alpha_{i}\right)\left(a_{i}-c_{i}\right) \\
& \left(Q_{i}-Q_{i}\right)_{\alpha}^{+}=\left(1-\alpha_{i}\right)\left(c_{i}-\hat{a}_{i}\right)+\alpha_{i}\left(b_{i}-b_{i}\right)
\end{aligned}
$$

From Definition 2, we know that $\left|Q_{i}-Q_{i}\right|$ is also a fuzzy number. There are four different cases which cause all kinds of results as follows.

Case A: If $\left(Q_{i}-Q_{i}\right)_{\alpha}^{-}>0$, the following formulas are available

$$
\begin{aligned}
& \left|Q_{i}-Q_{i}\right|_{\alpha}^{-}=\left(Q_{i}-Q_{i}\right)_{\alpha}^{-} \\
& \left|Q_{i}-Q_{i}\right|_{\alpha}^{+}=\left(Q_{i}-Q_{i}\right)_{\alpha}^{+},
\end{aligned}
$$

which result in

$$
\begin{aligned}
& \operatorname{POS}\left(\left|Q_{i}-\hat{Q}_{i}\right| \leq f_{i}\right) \geq \alpha_{i} \Leftrightarrow \\
& \alpha_{i}\left(b_{i}-b_{i}\right)+\left(1-\alpha_{i}\right)\left(a_{i}-c_{i}\right) \\
& \leq f_{i} \leq\left(1-\alpha_{i}\right)\left(c_{i}-a_{i}\right)+\alpha_{i}\left(b_{i}-b_{i}\right)
\end{aligned}
$$

Case B: If $\left(Q_{i}-Q_{i}\right)_{\alpha}^{+}<0$, then we have

$$
\begin{aligned}
& \left|Q_{i}-Q_{i}\right|_{\alpha}^{-}=-\left(Q_{i}-Q_{i}\right)_{\alpha}^{+} \\
& \left|Q_{i}-Q_{i}\right|_{\alpha}^{+}=-\left(Q_{i}-Q_{i}\right)_{\alpha}^{-}
\end{aligned}
$$

meanwhile, the followed formula obtained

$$
\begin{aligned}
& \operatorname{POS}\left(\left|Q_{i}-Q_{i}\right| \leq f_{i}\right) \geq \alpha_{i} \Leftrightarrow \\
& \left(b_{i}-b_{i}\right) \alpha_{i}+\left(1-\alpha_{i}\right)\left(a_{i}-c_{i}\right) \\
& \leq f_{i} \leq\left(b_{i}-b_{i}\right) \alpha_{i}+\left(1-\alpha_{i}\right)\left(c_{i}-a_{i}\right)
\end{aligned}
$$

Case C: If $\quad\left(Q_{i}-Q_{i}\right)_{\alpha}^{-}<0<\left(Q_{i}-Q_{i}\right)_{\alpha}^{+}$, and

$$
\left|\left(Q_{i}-Q_{i}\right)_{\alpha}^{-}\right|<\left|\left(Q_{i}-Q_{i}\right)_{\alpha}^{+}\right|
$$

we get the following formulas

$$
\begin{gathered}
\left|Q_{i}-Q_{i}\right|_{\alpha}^{-}=0 \\
\left|Q_{i}-Q_{i}\right|_{\alpha}^{+}=\left(Q_{i}-Q_{i}\right)_{\alpha}^{+}
\end{gathered}
$$

as well as

$$
\begin{aligned}
& \operatorname{POS}\left(\left|Q_{i}-Q_{i}\right| \leq f_{i}\right) \geq \alpha_{i} \Leftrightarrow \\
& 0 \leq f_{i} \leq\left(1-\alpha_{i}\right)\left(c_{i}-a_{i}\right)+\alpha_{i}\left(b_{i}-b_{i}\right)
\end{aligned}
$$

Case D: If $\left(Q_{i}-Q_{i}\right)_{\alpha}^{-}<0<\left(Q_{i}-Q_{i}\right)_{\alpha}^{+}, \quad$ and $\left|\left(Q_{i}-Q_{i}\right)_{\alpha}^{-}\right|>\left|\left(Q_{i}-Q_{i}\right)_{\alpha}^{+}\right|$then we obtain the formulas. It is shown as

$$
\begin{gathered}
\left|Q_{i}-Q_{i}\right|_{\alpha}^{-}=0 \\
\left|Q_{i}-Q_{i}\right|_{\alpha}^{+}=-\left(Q_{i}-Q_{i}\right)_{\alpha}^{+}
\end{gathered}
$$

and the followed formula is available

$$
\begin{aligned}
& \operatorname{POS}\left(\left|Q_{i}-Q_{i}\right| \leq f_{i}\right) \geq \alpha_{i} \\
& \Leftrightarrow 0 \leq f_{i} \leq\left(b_{i}-b_{i}\right) \alpha_{i}+\left(1-\alpha_{i}\right)\left(c_{i}-a_{i}\right)
\end{aligned}
$$

\section{E. Defuzzification}

An important concept regarding the application of fuzzy numbers is defuzzification, which transform a fuzzy number into a crisp value. Many different methods for this transformation can be utilized. The most commonly used defuzzification method is the centroid defuzzification method, which is also known as the center of gravity (COG) or center of area defuzzification. The defuzzification method can be presented as follow:

$$
\bar{Q}(\tilde{A})=\frac{\int_{a}^{d} Q \mu \tilde{A}(Q) d Q}{\int_{a}^{d} \mu \tilde{A}(Q) d Q}
$$

where $\bar{Q}(\tilde{A})$ is the defuzzified value. For trapezoidal fuzzy number $\left(a_{1}, a_{2}, a_{3}, a_{4}\right)$, the centroid-based defuzzified value turns out to be:

$$
\bar{Q}(\tilde{A})=\frac{1}{3}\left[a_{1}+a_{2}+a_{3}+a_{4}-\frac{a_{4} a_{3}-a_{1} a_{2}}{\left(a_{4}+a_{3}\right)-\left(a_{1}+a_{2}\right)}\right]
$$

Especially, when $a_{2}=a_{3}$, the above formula becomes as follows.

$$
\bar{Q}(\tilde{A})=\frac{1}{3}\left[a_{1}+a_{2}+a_{4}\right]
$$

It is the defuzzification formula of triangular fuzzy number $\left(a_{1}, a_{2}, a_{3}\right)$, which is utilized in this paper.

Step 1: Compute the member function $\mu_{\tilde{A}}$ of $\tilde{A}=\left(a_{1}, a_{2}, a_{3}\right)$. 
Step 2: Compute $\bar{Q}(\tilde{A})$ according to Step 1 .

Step 3: Output $\bar{Q}(\tilde{A})$, end.

\section{RESULT AND DISCUSSION}

As maintained above, the fuzzy $\operatorname{GM}(1,1)$ predicting model is employed for $\mathrm{CO}_{2}$ emissions prediction in Leshan city.

\section{A. Computing and Result}

Stage 1. In order to get the triangular fuzzy number of $\mathrm{CO}_{2}$ emissions, we first make fuzzification on it for the past 12 years. We assume that

$$
\begin{gathered}
Q_{0}(k)=\left(Q_{k}^{-}, Q_{k}, Q_{k}^{+}\right), Q_{k}-Q_{k}^{-}=\alpha, \\
Q_{k}^{+}-Q_{k}=\beta, \alpha=\beta=v
\end{gathered}
$$

where the number $v$ is gotten by experts of statistics department based on their experience and the current situation of local economic development.

According to the above methods, we make fuzzification on the original real data, and get the triangular fuzzy number of $\mathrm{CO}_{2}$ emissions in Leshan city.

Stage 2. The prediction model is employed to calculate the data above and obtain the predicted value of $\mathrm{CO}_{2}$ emissions in Leshan city.

Stage 3. Defuzzification on the predicted value use the centroid defuzzification method:

$$
\sum \mathrm{O}_{2}{ }^{-} \bar{Q}\left(\AA \rho=\frac{1}{3}\left[\hat{Q}^{-}+\hat{Q}^{-}+\hat{Q}^{+}\right]\right.
$$

Then, input the triangular fuzzy number of the predicted value $\hat{Q}$ into formula, get the defuzzificted value.

\section{B. Reason and Trend Analysis}

In this section, we predict the $\mathrm{CO}_{2}$ emissions in Leshan city region by using the fuzzy gray prediction model, and get a series of data in Fig. 4-Fig. 9. Now, we analyze these data, meanwhile, try to seek reasons, and find trend based the reasons. In addition, the law about the change of $\mathrm{CO}_{2}$ emissions will be sought out and make appropriate policies and measures available.

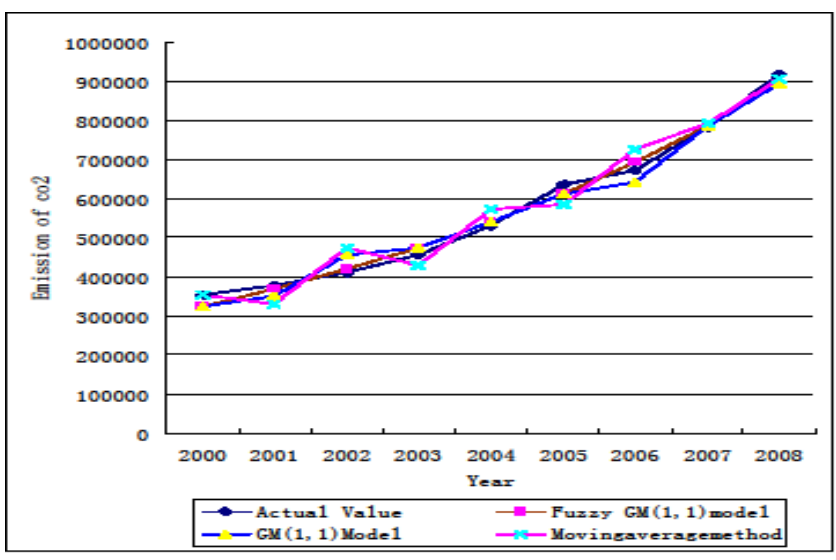

Fig. 4. $\sum \mathrm{O}_{2}{ }^{-}$emissions from 2001 to 2011

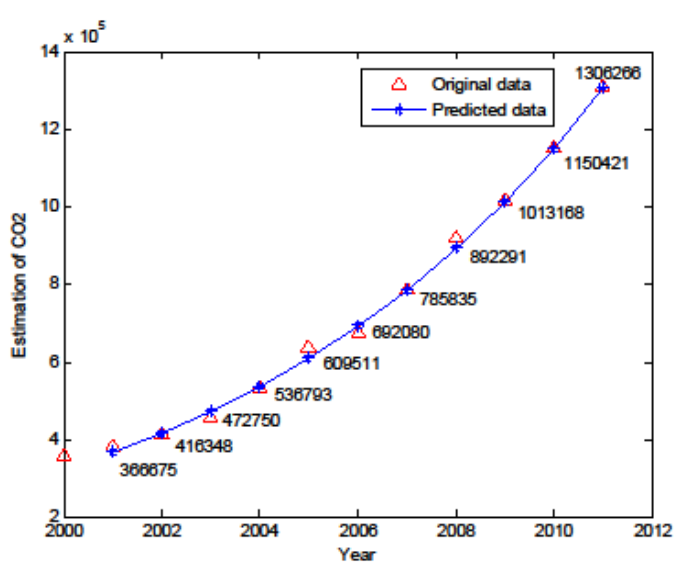

Fig. 5. $\mathrm{EO}_{2}{ }^{-}$emissions from 2012 to 2020.

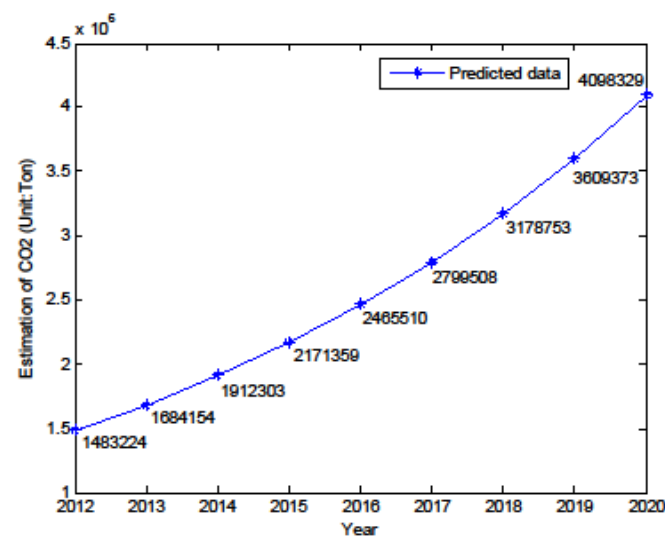

Fig. 6. $\mathrm{EO}_{2}{ }^{-}$emissions from 2001 to 2011.

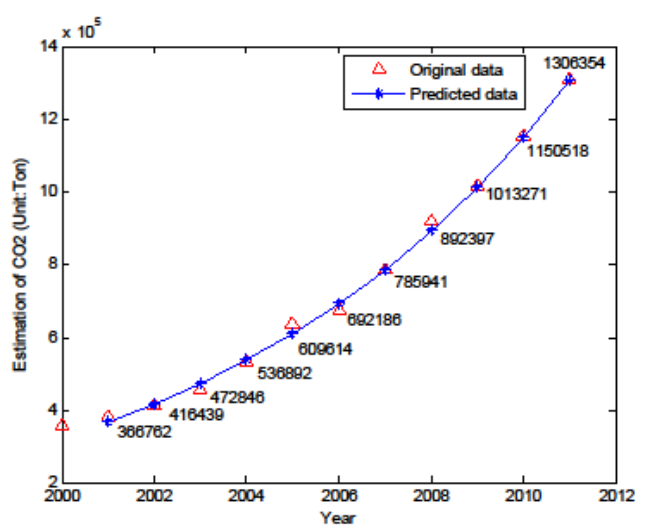

Fig. 7. $\mathrm{E}_{2}{ }^{-}$emissions from 2012 to 2020 .

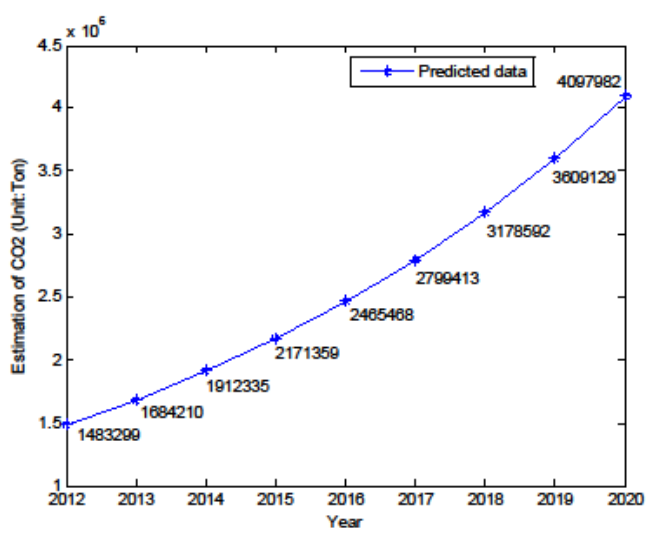

Fig. 8. $\sum_{\mathrm{O}_{2}}{ }^{-}$emissions from 2001 to 2011. 


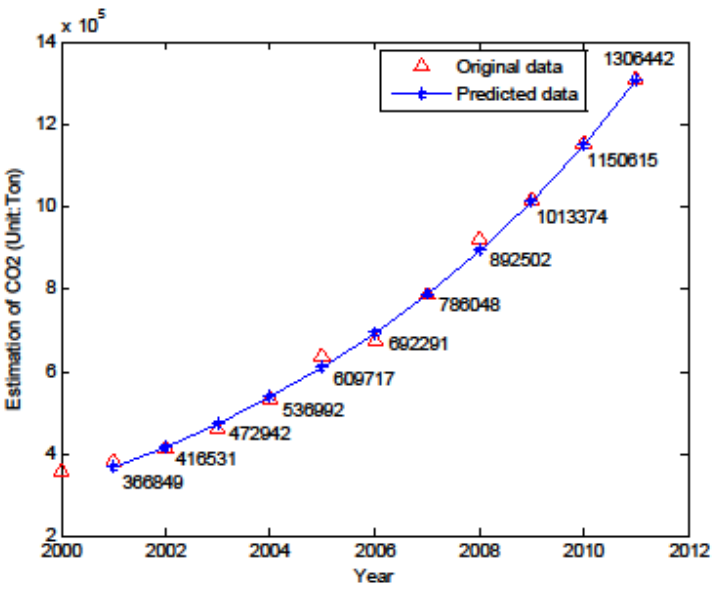

Fig. 9. $\sum_{\mathrm{O}_{2}}{ }^{-}$emissions from 2012 to 2020 .

Firstly, it can be seen from the figure that the $\mathrm{CO}_{2}$ emissions are increasing. In the past 12 year, due to advances in science, technology and the gradual improvement of national economic policy, and tourism was no longer the only developed industry in Leshan city. A number of industrial enterprises have been introduced, which lead to the increase of GHG emissions, especially the sharp growth in $\mathrm{CO}_{2}$ emissions.

Secondly, as it is shown in the figure, the location of historical data points and forecasted data points on the curve is consistent, which shows that the actual data and forecasted data is in a high degree of data fit. Meanwhile, it illustrates the high accuracy of the model, as well as of the forecast data.

Thirdly, we can see from the figure in the next few years, the increase rate of $\mathrm{CO}_{2}$ emissions in Leshan city is still high. This means that the environment pollution of the world double inheritance areas will be further deepened. Due to the world double inheritance regions' uniqueness and irreplaceability, environmental protection efforts in these regions is particularly imperative.

\section{Contrast Analysis}

Just as introduced above, we use the fuzzy possibility method and the data get form the fuzzy $\operatorname{GM}(1,1)$ check the model. According to the experts' advice, we suppose $f=\frac{1}{3}\left[Q^{-}+Q+Q^{+}\right]$and $\alpha=0.9$, then put the data into formula, where $\alpha 10224+(1-\alpha) 8082 \leq f \leq(1-\alpha) 12391+\alpha 10224$ $f=\frac{1}{3}\left[Q^{-}+Q+Q^{+}\right]$and $\alpha=0.9$ a, this formula is available. Similarly, we put all the data into this formula, and results are all right which shown fuzzy $\operatorname{GM}(1,1)$ model meet a test.

Furthermore, the mean absolute error method (MAE) is employed to make contrast analysis on the result obtained from $\operatorname{GM}(1,1)$ model, fuzzy $\operatorname{GM}(1,1)$ model as well as Moving average method, and the result shown in Table II. Table II and Fig. 10 shows that the MAE of Moving average method is larger than the other two methods, and the MAE of fuzzy $\operatorname{GM}(1,1)$ model is the least. Consequently, the accuracy of fuzzy $\operatorname{GM}(1,1)$ model is higher than the other two kinds method, which prove our method is applicable.

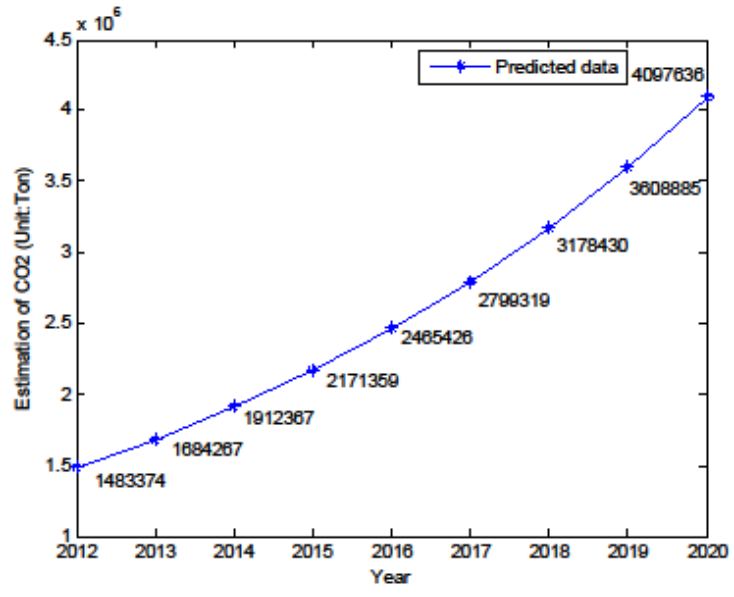

Fig. 10. Trend of actual value and forecast values (unit:Ton).

TABLE II: THE RESULT OF CONTRAST ANALYSIS

\begin{tabular}{cc}
\hline Prediction method & MAE \\
\hline Movingaveragemethod & 2.39 \\
GM $(1,1)$ model & 2.36 \\
Fuzzy GM $(1,1)$ model & 2.33 \\
\hline
\end{tabular}

\section{Countermeasures and Suggestions}

In view of these circumstances which lead to the increase of emissions, appropriate measures must be taken to reduce the $\mathrm{CO}_{2}$ emissions.

Firstly, it is important to control increment, and optimize the industry structure. In addition, it is also necessary to control the gas-guzzling and high pollution industries should be prevented from growing rapidly. What's more, increasing the investment and implementing the key projects fully. We should speed up the implementation on the ten major energy conservation projects and water saving projects.

Secondly, relying on science and technology to accelerate technological development and generalization is feasible. Besides, in order to lay a solid foundation the od energy saving management and emission reduction should be enhanced.

Thirdly, it is essential to improve the legal system, and strengthen the supervision, inspection and enforcement. At the same time, we should pay more attention to perfection of the policies and the formation of the incentive and restraint mechanisms. Finally, in order to strengthen the publicity, and enhance the consciousness of national citizen. Such activities are practicable.

\section{CONCLUSION}

In this study, fuzzy grey method is employed to make prediction based on the date of $\mathrm{CO}_{2}$ emissions in the past 12 years of Leshan city, the $\mathrm{CO}_{2}$ emissions date of the nest eight years are obtained. Fuzzy possibility check and contrasted analysis show the accuracy of the model is quite high, providing a scientific basis for policy makers.

In a word, the established fuzzy grey predict modeling can accurately predict the $\mathrm{CO}_{2}$ emissions date, giving support for policy makers in Leshan city and other the world natural and cultural heritage areas. 


\section{ACKNOWLEDGMENT}

This research was supported by the Key Program of NSFC (Grant No. 70831005), and the Key Project of China Petroleum and Chemical Corporation (Grant No. GJ-73-0706).

\section{REFERENCES}

[1] Y. Fan, Q. M. Liang, Y. M. Wei, and N. Okada, "A model for China's energy requirements and $\mathrm{CO}_{2}$ emissions analysis," Environmental Modelling \& Software, vol. 22, no. 3, pp. 378-393, 2007.

[2] J. I. Arar and D. Southgate, "Valuating $\mathrm{CO}_{2}$ reduction strategies in the US," Ecological Modelling, vol. 220, no. 4, pp, 582-588, 2009.

[3] M. A. L. Caetano, D. F. M. Gherardi, and T. Yoneyama, "Optimal resource management control for $\mathrm{CO}_{2}$ emission and reduction of the greenhouse effect," Ecological Modelling, vol. 213, no. 1, pp. 119-126, 2008.

[4] A. W. King, R. V. O'Neill, and D. L. DeAngelis, "Using ecosystem models to predict regional $\mathrm{CO}_{2}$ exchange between the atmosphere and the terrestrial biosphere," Global Biogeochemical Cycles, vol. 3, no. 4, pp. 337-361, 1989 .

[5] R. B. Bacastow and C. D. Keeling, "Models to predict future atmospheric $\mathrm{CO}_{2}$ concentrations," in Proc. Workshop on the Global Effects of Carbon Dioxide from Fossil Fuels, pp.72-90, 1979.

[6] J. W. Raich and C. S. Potter, "Global patterns of carbon dioxide emissions from soils," Global Biogeochemical Cycles, vol. 9, no. 1, pp. 23-36, 1995
[7] Y. S. Kim, W. Y. Choi, J. H. Jang, K. P. Yoo, and C. S. Lee, "Solubility measurement and prediction of carbon dioxide in ionic liquids," Fluid Phase Equilibria, vol. 228, pp. 439-445, 2005.

[8] S. H. Park, K. B. Lee, J. C. Hyun, and S. H. Kim, "Correlation and prediction of the solubility of carbon dioxide in aqueous alkanolamine and mixed alkanolamine solutions," Industrial \& Engineering Chemistry Research, vol. 41, no. 6, pp.1658-1665, 2002.

[9] J. L. Deng, "Introduction to grey system theory," The Journal of Grey System, vol. 1, no. 1, pp. 1-24, 1989.

[10] H. J. Zimmermann, Fuzzy Set Theory and Its Applications, Springer Netherlands, 2001

[11] L. A. Zadeh, "Fuzzy sets," Information and Control, vol. 8, no. 3, pp. 338-353, 1965.

[12] J. P. Xu and X. Zhou, "Fuzzy-like multiple objective decision making," Studies in Fuzziness and Soft Computing, vol. 263, Springer-Verlag Berlin Heidelberg, 2011

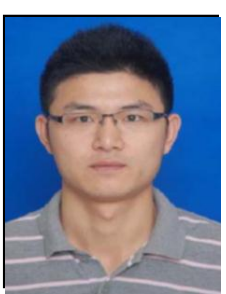

Zhiyi Meng was born in 1987. He received his M.S degree in Sichuan University. He is currently a Ph.D candidate at Sichuan University. His research interests include systems engineering and information system. 